\title{
Silk fibroins modify the atmospheric low temperature plasma-treated poly (3-hydroxybutyrate-co-3-hydroxyhexanoate) film for the application of cardiovascular tissue engineering
}

\author{
Huaxiao Yang ${ }^{1}$, Min Sun ${ }^{1}$, Ping Zhou ${ }^{1}$, Luanfeng Pan $^{2}$, Chungen $\mathrm{Wu}^{2}$ \\ ${ }^{1}$ The Key Laboratory of Molecular Engineering of Polymers Ministry of Education, Department of Macromolecular Science, Fudan \\ University Shanghai, China; \\ ${ }^{2}$ Laboratory of Molecular Biology Shanghai Medical College, Fudan University Shanghai, China. \\ Email: pingzhou@fudan.edu.cn; lfpan@shum.edu.cn
}

Received 22 Octorber 2010; revised 26 Octorber 2010; accepted 29 Octorber 2010.

\begin{abstract}
Tissue engineered scaffold is one of the hopeful therapies for the patients with organ or tissue damages. The key element for a tissue engineered scaffold material is high biocompatibility. Herein the poly (3hydroxybutyrate-co-3-hydroxyhexanoate) (PHBHHx) film was irradiated by the low temperature atmospheric plasma and then coated by the silk fibroins (SF). After plasma treatment, the surface of PHBHHx film became rougher and more hydrophilic than that of original film. The experiment of PHBHHx flushed by phosphate buffer solution (PBS) proves that the coated SF shows stronger immobilization on the plasma-treated film than that on the untreated film. The cell viability assay demonstrates that SF-coated PHBHHx films treated by the plasma significantly supports the proliferation and growth of the human smooth muscle cells (HSMCs). Furthermore, the scanning electron microscopy and hemotoylin and eosin (HE) staining show that HSMCs formed a cell sub-monolayer and secreted a large amount of extracellular matrix (ECM) on the films after one week's culture. The silk fibroins modify the plasmatreated PHBHHx film, providing a material potentially applicable in the cardiovascular tissue engineering.
\end{abstract}

Keywords: Biocompatible; Cardiovascular Tissue Engineering; Low Temperature Plasma; Poly(3-Hydroxybutyrate-Co-3-Hydroxyhexanoate) (PHBHHx); Silk Fibroin

\section{INTRODUCTION}

Tissue engineering (TE) [1] involves many issues in- cluding scaffold design [2-4], cell culture and differenttiation [5], bio-interfacial interaction between cell and material [6-8], and tissue regenerative induction [9]. Tissue engineering opens a hopeful door for the patients who need organs or tissues repaired, replaced, or regenerated [10]. Apparently, one of the most significant issues of TE is the scaffold design. A sound scaffold not only supports the cell adhering and proliferating at the early stage in vitro [8] but also maintains the primary functions as an artificial organ in vivo [11]. In the past decade, the bio-synthetic biodegradable polyesters, polyhydroxyalkanoates (PHAs) [2,12,13], have attracted many attentions and were widely investigated for the applications in the biomedical engineering, such as the scaffold of poly(3-hydroxybutyrate-co-3-hydroxyhexanoate) (PHBHHx)/hydroxyapatite for bone reconstructtion [14], the biocompatibility of PHBHHx for bone marrow stromal cells in vitro [15], the microspheres of PHBHHx for the drug controlled release [16,17], the nanofibrous matrix of PHA for the better cell growth [4] and so on, because the PHAs have adjustable mechanical properties and biodegrading rates by varying the content $x$ of hydroxyhexanoate $(\mathrm{HH})$ in PHBHHx $[2,12,18,19]$.

However, like most of polyesters, PHBHHx is also fair hydrophobic and not so proper for the cell adhesion and growth, and has no active functional groups for the attachment of biomolecules [12,20]. As the results, some methods have been applied to improve the PHBHHx surface hydrophilicity and biocompatibility, for instance, the maleic anhydride was grafted onto the $\mathrm{PHBHHx}$ to form the maleated PHBHHx, which showed the improved biocompatibility, suitable mechanical properties as well as the accelerated biodegradation [21]. In addition, significant increase in the growth of the fibroblasts 
L929 was also observed on the films prepared by the ultraviolet-radiated PHBHHx powders [22]. Our group modified the PHBHHx films and porous scaffolds by coating silk fibroin (SF), demonstrating that the SFcoating highly improved the hydrophilicity of the scaffold and proliferations of cardiovascular related cells seeded on them $[8,23,24]$.

Low temperature plasma (LTP) is an effective method for modifying the material surface properties including wettability, topography and surface charge states. It can penetrate the material in depth of 10-100 $\AA$ without damaging the bulk material [25-28], therefore LTP exhibits the comprehensive applications in the surface modification of polymeric scaffolds [25,29,30]. In addition, LTP is also used to introduce some specific elements or functional groups onto the surface of polymer, such as protein [27], peptide [31], and polysaccharide [32]. Among the plasma techniques, dielectric barrier discharge (DBD) presents the advantages as compared to the conventional techniques which use electron, ion and photon beams to bomb the surface under vacuum condition. Using the DBD technique, the discharge can be operated at normal atmospheric pressure with various gases, e.g. corrosive gases, dry and humid gas [33]. More importantly, many functional groups can be intro- duced and grafted onto the surface after plasma treat- ment by altering working gas [34], for example, - $\mathrm{COOH}$ group can be grafted by $\mathrm{CO}_{2}$ plasma, $-\mathrm{NH}_{2}$ or $-\mathrm{NH}$ - group can be grafted by $\mathrm{NH}_{3}$ plasma, - $\mathrm{OH}$ and -CO- groups can be grafted by water $/ \mathrm{O}_{2}$ or air plasma. These grafted functional groups can improve the hydrophilicity of the material surface [30].

In present work, to have the SF interaction with the PHBHHx surface stronger and improve the biocompatibility of the PHBHHx film, the surface of PHBHHx films was irradiated by DBD plasma at atmospheric pressure with argon as working gas, and then coated by SF. The optimal plasma irradiation time was evaluated by the water contact angle. The experiment of PHBHHx flushed by phosphate buffer solution (PBS) under the rate of physiologic blood flow was designed to test the strength of SF immobilization on the surface of plasmatreated PHBHHx films. The proliferation and morphologies of the human smooth muscle cells (HSMCs) cultured on the films were also investigated.

\section{MATERIALS AND METHODS}

\subsection{Materials}

PHBHHx powder $(\mathrm{Mw}=100,000, \mathrm{x}=12 \mathrm{~mol} \%$ for the HH content) was kindly donated by Prof. Guo-Qiang Chen in Tsinghua University, China. The PHBHHx was purified by dissolving it in the dichloromethane $\left(\mathrm{CH}_{2} \mathrm{Cl}_{2}\right)$ solution, and fully refluxed at $40^{\circ} \mathrm{C}$ for half an hour, and then filtered through a piece of qualitative filter paper, and re-precipitated in n-hexane solution. The resultant solid sample was dried at room temperature for more than 24 hours, and stored in the desiccator for later use. About $0.6 \mathrm{~g}$ purified PHBHHx powder was dissolved in $10 \mathrm{~mL} \mathrm{CH}_{2} \mathrm{Cl}_{2}$, and then the solution was cast on a glass plate in diameter of $60 \mathrm{~mm}$ in fume hood at room temperature for 24 hours. A film in thickness of $97 \pm 1 \mu \mathrm{m}$ was obtained.

The regenerated silk fibroin (SF) solution was prepared following the report of Mei et al [8]. In brief, raw Bombyx mori silk was degummed twice with $0.5 \mathrm{wt} \%$ $\mathrm{NaCO}_{3}$ solutions at $100^{\circ} \mathrm{C}$ for 1 hour and then washed with deionized water. The degummed silk was dissolved in $9.3 \mathrm{~mol} / \mathrm{L} \mathrm{LiBr}$ solution at room temperature. After dialysis against the deionized water for more than three days to remove $\mathrm{LiBr}$ salt, the solution was filtered to remove the impurities. The regenerated silk fibroin solution with concentration of $\sim 2 \%(\mathrm{w} / \mathrm{v})$ was obtained and further diluted to the concentration of $1 \%(\mathrm{w} / \mathrm{v})$.

For easily and quantitatively probing the silk fibroin content in the dilute solution, the silk fibroin was labeled by fluorescein isothiocyanate (FITC) which can be detected by the fluorescent spectrometer. The FITC-labeled SF solution was prepared as follows: $20 \mathrm{~mL} 1 \%(\mathrm{w} / \mathrm{v})$ SF solution was adjusted to $\mathrm{pH}=7.0$ by $0.01 \mathrm{~mol} / \mathrm{L}$ $\mathrm{NaOH}$ solution, then $0.1 \mathrm{mg}$ FITC powder (purity of $90 \%$, J\&K Chemical, Sweden) was added into the solution. The solution was stirred at $20^{\circ} \mathrm{C}$ for 2 hours, and then stored at $4^{\circ} \mathrm{C}$ for overnight, and then dialyzed against deionized water for four days. The water was refreshed every 3 or 4 hours to remove excessive FITC in the solution. The whole process was operated in a dim room. The final concentration of FITC-labeled SF solution was about $0.94 \%(\mathrm{w} / \mathrm{v})$ and the solution was stored in the refrigerator at $4^{\circ} \mathrm{C}$ for later use.

\subsection{Surface Modification of PHBHHx Film}

\subsubsection{Atmospheric Plasma Treatment}

The plasma treatment was carried out by a low temperature atmospheric plasma generator (CTP-2000K, Nanjing Shuman Ltd. Company, China) shown in Figure 1. The quartz DBD generator was operated at a frequency of $20 \mathrm{MHz}$. The sample was irradiated by the plasma generated between two plate electrodes with diameter of $4 \mathrm{~cm}$ and distance of $2 \mathrm{~cm}$ (shown in Figure 1). The PHBHHx film in diameter of $1.5 \mathrm{~cm}$ was put on the ground electrode in the quartz chamber. The argon gas (purity of 99.9\%) was conducted into a chamber which was connected to the air and the plasma was generated by the power of $50 \mathrm{~W}$ at atmospheric pressure. The plasma irradiating time was changed from 1 to $10 \mathrm{~min}$ - 


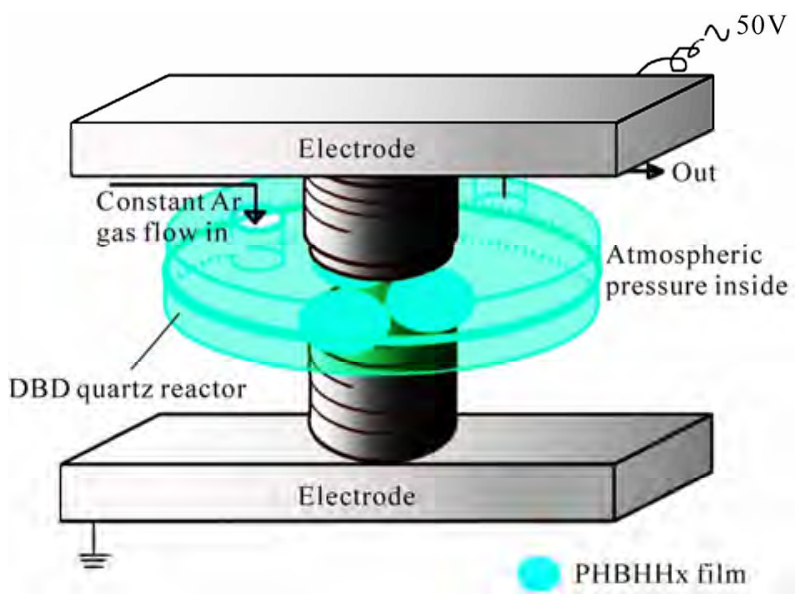

Figure 1. Schematic graph of low temperature atmospheric plasma generating device.(C)

utes to determine an optimal irradiating time which was evaluated by the water contact angle of the film. The smaller water contact angle indicates the better wettability of the PHBHHx films.

\subsubsection{Morphology measurement by SEM}

The morphologies of pristine and plasma treated PHBHHx films were measured by the scanning electron microscopy (SEM). Briefly, sample was sputter-coated by an ultrathin gold layer for 60 seconds with current of $10 \mathrm{~mA}$, and then observed by SEM (TS 5136MM, Tescan vega, Czech) with an accelerating voltage of $20 \mathrm{kV}$.

\subsubsection{Measurement of Water Contact Angle}

The water contact angle was measured by OCA 15 plus equipment (Data Physics, Germany) packaged with SCA-20 software. A drop of $1.5 \mu \mathrm{L}$ of distilled water was dropped onto the sample surface and its contact angle $(\theta)$ was measured within 10 seconds. The contact angle was mean of the ten values.

\subsubsection{Silk Fibroins Immobilization}

The PHBHHx films were immediately immersed into $1 \%(\mathrm{w} / \mathrm{v}) \mathrm{SF}$ solution for $30 \mathrm{~min}$ after plasma treatment, and then freeze-dried overnight under vacuum of 0.1 torr at $-50^{\circ} \mathrm{C}$. At the same time, PHBHHx film without plasma treatment was also immersed into $1 \%(\mathrm{w} / \mathrm{v}) \mathrm{SF}$ solution for $30 \mathrm{~min}$ and freeze-dried as control.

\subsubsection{Test of the Strength of SF Immobilization on the Plasma Treated PHBHHx Film}

The strength of SF immobilization on the plasma treated PHBHHx film was tested by flushing the sample with PBS solution. In this experiment, both of the SF-coated PHBHHx films with and without plasma treatment were flushed by recycled $90 \mathrm{~mL}$ PBS buffer $(\mathrm{pH}=7.4)$ for 30 min at the flow rate of $55 \mathrm{~mL} / \mathrm{min}$ which mimicked the physiologic blood fluid in vivo. During flushing, $2 \mathrm{~mL}$ flushed solution was sampled every 10 min for the fluorescent measurement. The content of the flushed SF in the PBS solution was measured by the FITC fluorescent at $520 \mathrm{~nm}$ (FLS 920, Edinburgh Instrument, Switzerland) where FITC was labeled on the SF.

\subsection{Human Smooth Muscle Cell (HSMCs) Isolation and Cells Culture in Vitro}

HSMCs were isolated from the human umbilical artery obtained from Shanghai No.1 Hospital for the Protection of Mother and Baby's Health and puerperal informed consent, and the method of isolation was referred to the literature [24]. In brief, a $25 \mathrm{~cm}$ length of human umbilical vein was cleaned with the sterile gauze to remove blood contaminations, and then the vascular tunica media layer was prudently separated from the vein. The layer was thoroughly washed with serum free Dulbecco's modified eagle medium (DMEM, Gibco, USA) to wipe off the portion of connective tissue, and then cut into ten pieces of tissues in $1 \mathrm{~mm}^{3}$. Those pieces of tissues were put on the bottom of culture flask (polystyrene, Corning, USA) in a proper distance between each other and cultured in an incubator with saturated humid air and $5 \% \mathrm{CO}_{2}$ for 12 hours at $37^{\circ} \mathrm{C}$ to allow the cells adhering and migrating in the flask. After most of tissues firmly attached on the bottom of flask, the culture medium (DMEM with 10\% (v/v) fetal bovine serum (FBS, Hyclone, USA), $100 \mathrm{U}$ streptomycin and $100 \mu \mathrm{g} / \mathrm{ml}$ penicillin) was added into the flask, just covering over the tissue pieces for continually culturing the cells in the incubator. The medium was refreshed every 2 days until the cells grew into the confluence by $80 \%$. The cells were gently digested with $0.25 \%$ trypsinase- $0.01 \%$ EDTA solution and passed into the next passage of cells for further culture. The cells within 5 to 8 passages were used in the study.

\subsection{Cells Seeded on the Studied Films}

HSMCs (passage of 5-8) were harvested by $0.25 \%$ trypsinase- $-0.01 \%$ EDTA and diluted to the concentration of $4.0 \times 10^{5}$ cells $/ \mathrm{mL}$. The cells were seeded on four types of PHBHHx films including PHBHHx film (P), plasma treated PHBHHx film (PP), SF-coated PHBHHx film (SP), and plasma treated PHBHHx film coated by SF (SPP). Those films were carefully put into 24-well culture plates $\left(\right.$ Coster $^{\mathrm{TM}}$ ) and sterilized in $75 \%$ (v/v) ethanol solution overnight, and then rinsed with PBS buffer to remove the ethanol and then irradiated by ultraviolet for 30 minutes for each side of the film. $100 \mu \mathrm{L} 4 \times 10^{5}$ cells/mL suspended cell solution was diluted in $300 \mu \mathrm{L}$ culture medium, and dropped onto each film. After the cells adhered on the films for $30 \mathrm{~min}$, extra $600 \mu \mathrm{L}$ culture medium was supplemented into each well. The cul- 
ture medium was refreshed every 2 days.

\subsection{Proliferation Analysis of HSMCs on the Studied Films}

\subsubsection{Mitochondrial Metabolic Activity of HSMCs on the Studied Films}

Mitochondrial metabolic activities of the HSMCs on the different types of films were evaluated by the MTT assay. MTT (3-(4, 5)-dimethylthiahiazo (-z-y1)-3, 5-diphenytetrazoliumromide (Merk, USA)) can be reduced by the succinate dehydrogenase when the mitochondrial of living cells interact with the MTT and form the purple formazan deposits. MTT assay can quantitatively assess the metabolic activity of cells when the HSMCs are cultured on the studied films. Briefly, after the culture medium in the plate well was removed, the cells on the films were rinsed by serum free DMEM medium three times and then incubated continually with $400 \mu \mathrm{L}$ DMEM medium and $40 \mu \mathrm{L}$ MTT solution ( $5 \mathrm{mg} / \mathrm{ml}$ in PBS) for $4 \mathrm{~h}$ under the humid condition at $37^{\circ} \mathrm{C}$, and then the insoluble purple formazan crystals were formed. The crystal was dissolved by $400 \mu \mathrm{L}$ dimethylsulfoxide (DMSO, Sigma, USA) and $200 \mu \mathrm{L}$ of the solution was transformed into a 96-well plate for the optical density (OD) measurement by a spectrophotometer (ELx800, BioTek, USA) at 565 $\mathrm{nm}$. The $200 \mu \mathrm{L}$ pure DMSO was used as blank control.

\subsubsection{HE Staining of HSMCs on the Studied Films}

Hemotoylin and eosin (HE) staining is a traditional, fast and reliable histological staining method to observe the cells. In this study, the HSMCs cultured on the studied films for 3, 5 and 7 days were rinsed with PBS buffer, and fixed by $4 \%$ polyformaldehyde buffer solution $(\mathrm{pH}$ $=7.4$ ) overnight at $4^{\circ} \mathrm{C}$, and then dehydrated by the ethanol with gradually increased concentration of 50, 60, 70, 80, 90, 95 and $100 \%(\mathrm{v} / \mathrm{v})$. The cells were stained with hemotoylin and eosin for 1 min respectively, and then gently washed with distilled water, and sealed with liquid paraffin. The cells were observed under the inverted light microscope (XDS-1B, Chongqing Optical Co., China) and recorded by a digital camera (Nikon D60).

\subsection{Morphology Observation of HSMCs Growing on the Studied Films}

Morphologies of the HSMCs cultured on the studied films for 3 and 7 days were observed by SEM. The HSMCs were fixed by $4 \%$ polyformaldehyde buffer solution ( $\mathrm{pH}=7.4)$ overnight at $4^{\circ} \mathrm{C}$, subsequently, dehydrated by the ethanol with gradually increased concentration of 50, 60, 70, 80, 90, 95 and $100 \%(\mathrm{v} / \mathrm{v})$, and then lyophilized overnight under vacuum of 0.1 torr at $-50^{\circ} \mathrm{C}$. Morphologies of the HSMCs cultured on the studied films were observed by SEM.

\subsection{Statistical Analysis}

Data were presented as means \pm SD (standard deviation). Three values were measured for every sample. Statistical comparisons were performed using ANOVA one-way method (Origin ${ }^{\mathrm{TM}}$ ). $P<0.05$ was considered statistically significant difference between two data groups.

\section{RESULTS AND DISCUSSION}

\subsection{Influence of Atmospheric Plasma Treatment on the Films}

In this study, the PHBHHx films were irradiated by low temperature atmospheric argon/air plasma for time of 1 , 2, 4, 6, 8 and 10 min. Plasma irradiation can break down the chemical bonds of the material to form free radicals, leading to the chemical composition rearranging and the surface roughening of polymeric matrix [33,35]. After the plasma treatment, the surface morphology of PHBHHx film became rougher than that of pristine film shown in Figure 2. The similar rough 'zones' were also observed when the PHBHHx film was irradiated by the ion implantation with fluence of $1 \times 10^{15}$ ions $/ \mathrm{cm}^{2}$ [20]. The hydrophilicity of the treated material surface was characterized by the water contact angles shown in Figure 3 . The results indicates that the water contact angles decreased from $90.3^{\circ}$ to $70.4^{\circ}$ when the films were irradiated by plasma from $1 \mathrm{~min}$ to $6 \mathrm{~min}$, and reached a constant value around $72^{\circ}$ when the irradiation time was longer. The decreases in the water contact angle are contributed to the plenty of hydrophilic groups, such as hydroxyl and carboxyl groups [25] formed on the surface of PHBHHx film after plasma treatment. The constant value of water contact angle is due to the hydrophilic functional groups saturated on the surfaces of films [27].

Considering the efficient treatment and no destruction for the bulk polymer, 6 min of the plasma irradiation time was selected as an optimum treating time in our experiment. The following reports will no longer mention this treating time any more.

\subsection{Strength of SF Immobilization on the Plasma Treated PHBHHx Films}

The SF-coated PHBHHx films that with and without plasma treatment were flushed by PBS buffer for $30 \mathrm{~min}$, and the FITC-labeled SF in the eluate was detected by the FITC fluorescence. The results are shown in Figure 4. The fluorescence increases as the flushing time increases for the SF-coated PHBHHx film without plasma treatment, indicating that the SF are easily desorbed from the SF-coated PHBHHx film, whilst very low fluorescence intensity and nearly no significant fluorescence change are found for the SF-coated PHBHHx film with plasma treatment, indicating that the SF were firmly adsorbed 


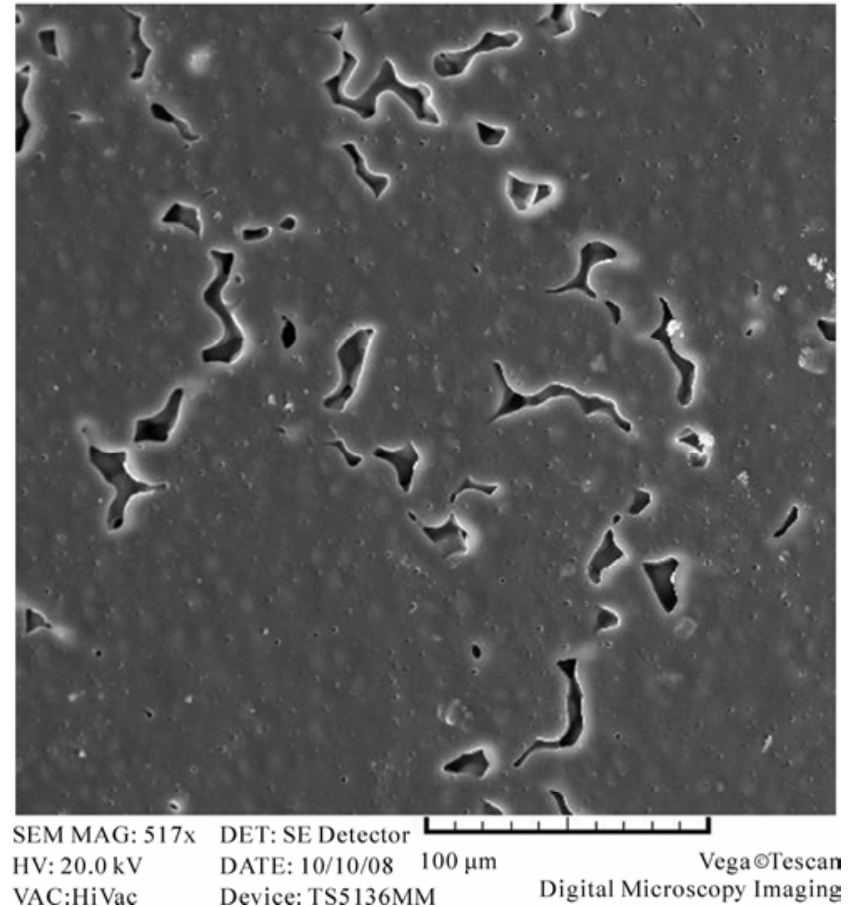

(a)

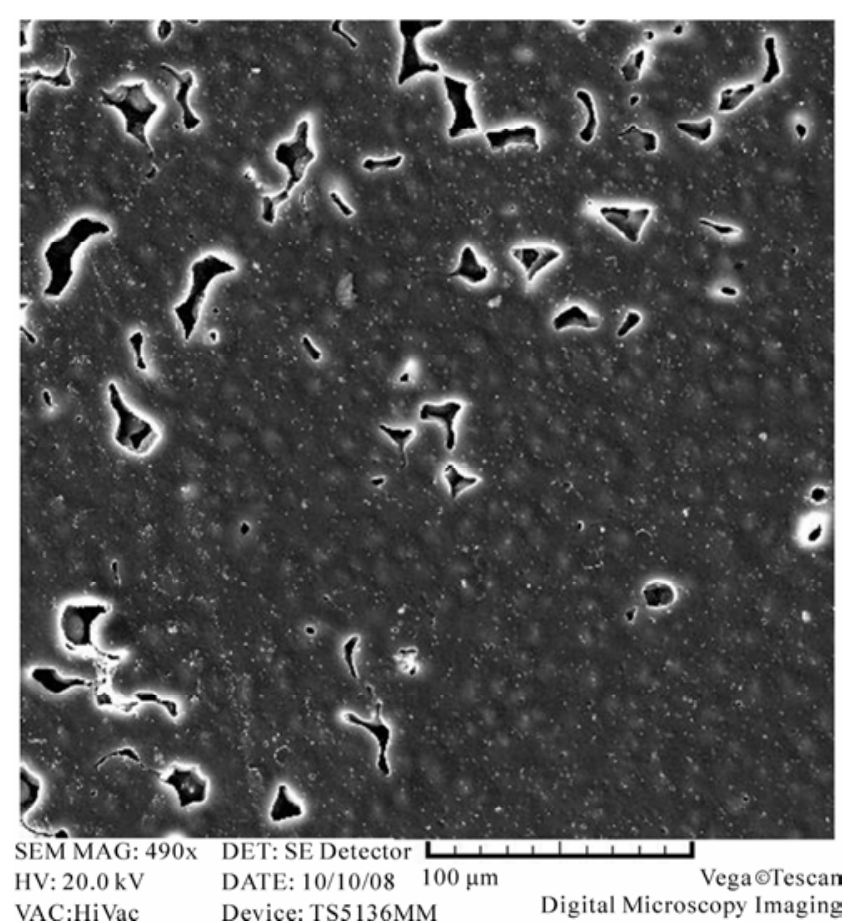

(b)

Figure 2. Comparison of the surface morphologies between pristine PHBHHx film (a) and plasma treated PHBHHx film for 6 min irradiation (b).

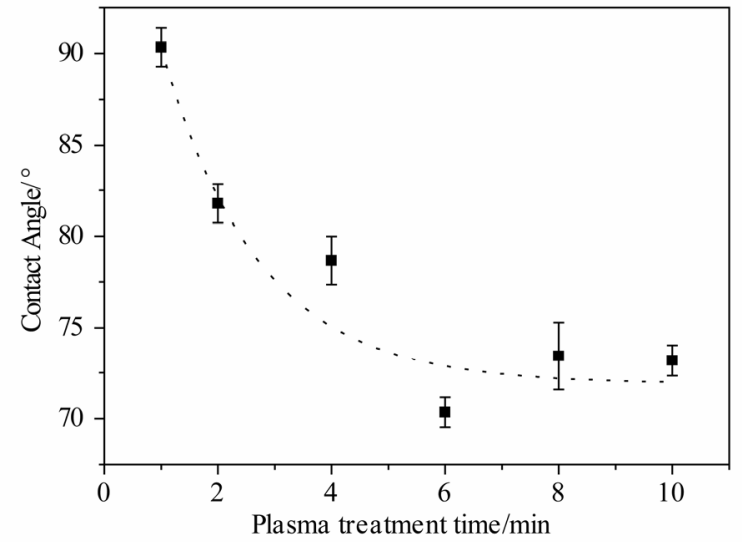

Figure 3. The water contact angles versus the plasma irradiation time for the PHBHHx film.

on the plasma-treated PHBHHx film even though under the physiologic recycle-flow flushing condition, possibly due to the hydrophilic chemical groups newly grafted on the surface. Therefore, the plasma-treated PHBHHx film is an ideal substrate for SF immobilization, and would be as a potential candidate material for the artificial blood vein to bear the high sheer force of blood flow in vivo.

\subsection{Growth and Proliferation of HSMCs on the Studied Films}

The growth and proliferation of HSMCs cultured on the

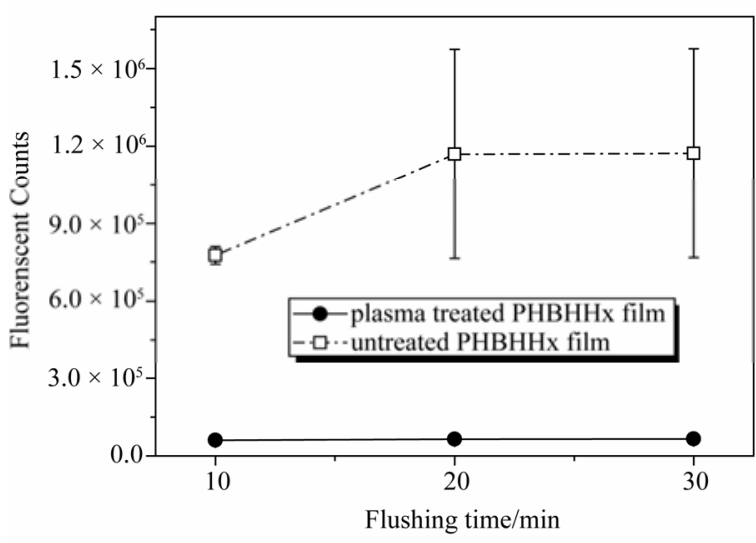

Figure 4. The fluorescents of FTIC-labeled SF in the PBS buffer solutions which were used to flush the SF-coated PHBHHx films with (in solid line) and without (in dash dot line) plasma treatment, respectively.

studied PHBHHx films up to 7 days were evaluated by MTT assay and HE staining method.

The MTT assay results are shown in Figure 5 which demonstrates the relative optical densities (OD) of four types of the studied films. The high OD value indicates the high cell viability. From the Figure 5, HSMCs seeded on the SF-coated PHBHHx film (SP) have the better cell viability than that on the PHBHHx film (P) after cultured for 7 days, which is consistent with our previous 


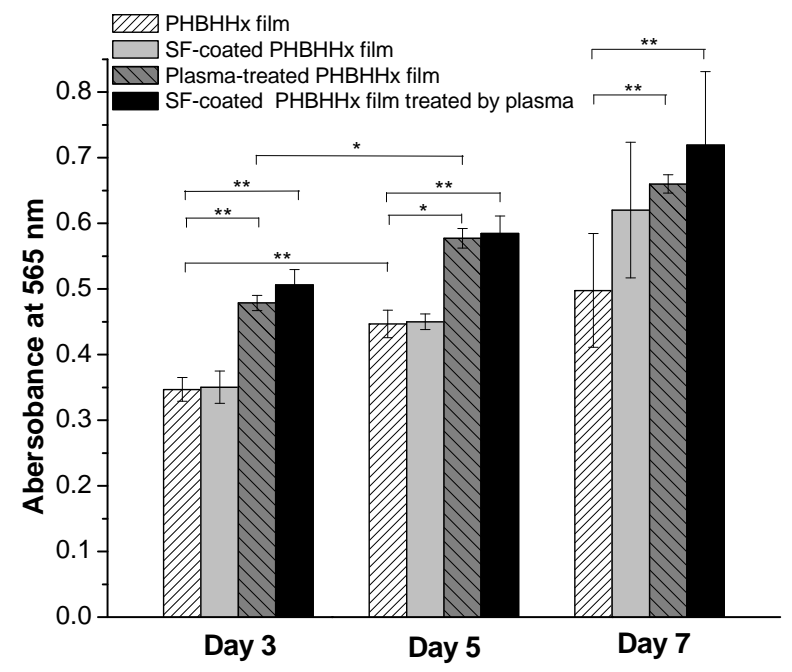

Figure 5. Cell vitality of HSMCs seeded on different types of PHBHHx films at day 3, 5 and 7 (*: $P<0.05$; **: $P<0.01, \mathrm{n}=$ 3).

results [7,24]. Furthermore, the plasma-treated PHBHHx films (PP) better support the growth and proliferation of the cells than the SF-coated film without plasma treatment. Apparently, HSMCs seeded on the SF-coated PHBHHx film treated by plasma (SPP) show the highest OD values during culture days, indicating that the film is suitable for the HSMCs adhering and proliferating. Armentano et al. reported that the oxygen-based plasma treated poly (L-lactide) (PLLA) film could improve the human marrow stromal cells (HMSCs) adhesion and growth [36]. Similarly, Shen et al found that the 3T3 fibroblasts on the $\mathrm{CO}_{2}$ plasma-treated poly (lactide-coglycolide) (PLGA) scaffold immobilized by the basic fibroblast growth factor (bFGF) showed the highest viability and the rapidest proliferation among the various PLGA scaffolds including those with and without plasma treatment and with bEGF immobilization [26]. These results substantially prove that plasma treatment for the material is an efficient method to improve the biocompatibility of the material.

Moreover, the shuffle-like HSMCs were observed by HE staining, where the cell nuclei are in dark purple and the cytoplasm in red shown in Figure 6. The HSMCs show the lower cell density on the P and SP films (Figure 6(a) and (b)) than that on the SPP films (Figure 6(d)) in 3 days culture, which show the highest cell den- sity among those studied films. In 7 days of cell culture, the proliferation of HSMCs on the pristine (Figure 6(a')) and plasma-treated PHBHHx films (Figure 6(c')) show the higher cell density than that in 3 days culture, and the HSMCs on the PP film (Figure 6(c')) not only expanded into the more cells than that on the P film (Figure 6(a')) but also started to form the cell network. Moreover, the HSMCs on the SP film (Figure 6(b')) and SPP film (Figure 6(d')) gradually form the larger cell sub-monolayer network than that on the PP film (Figure 6(c')).

\subsection{Morphologies of HSMCs Growing on the Studied Films}

The morphologies of HSMCs growing on the films of $\mathrm{P}$, SP, PP and SPP in 3 and 7 days culture were observed by SEM. In Figure 7, the HSMCs on the P film show a low cell density without typical shuffle-like morphology at day 3 (Figure 7(a)). Contrastively, the HSMCs growing on the SP, PP and SPP films show the higher cell density with the better cell shapes of shuffle-like morphology
P film

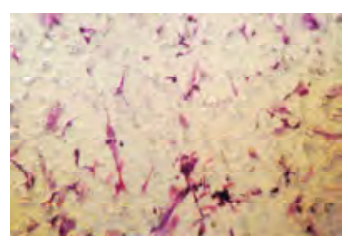

(a)

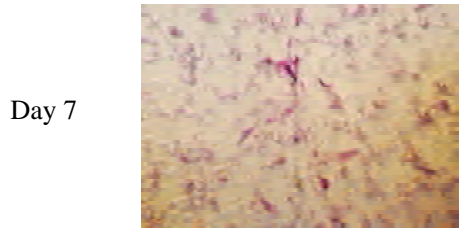

(a')
SP film

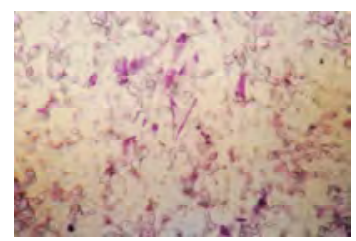

(b)

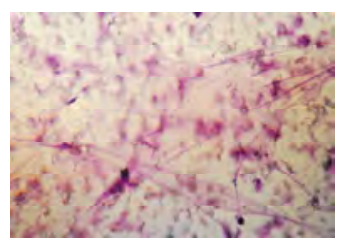

(b’)
PP film

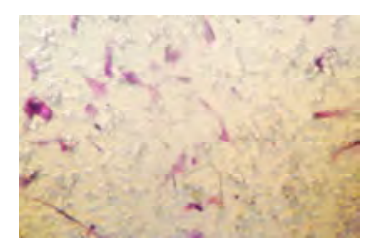

(c)

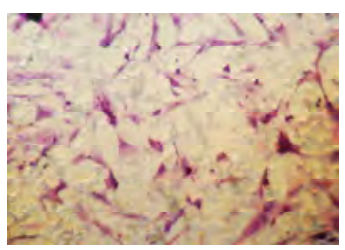

(c')
SPP film

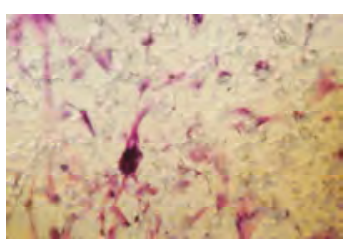

(d)

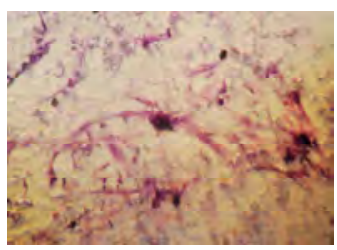

(d')

Figure 6. Images of HE-stained HSMCs cultured on four types of films at day 3 from (a) to (d) and day 7 from (a') to (d'). (a) and (a'): cells cultured on the pristine PHBHHx film (P film); (b) and (b’): cells cultured on the SF-coated PHBHHx film (SP film); (c) and (c'): cells cultured on the plasma-treated PHBHHx film (PP film); (d) and (d'): cells cultured on the SF-coated PHBHHx film treated by plasma (SPP film). The cell nuclei were stained in purple with hematoxylin and the cytoplasma was stained in red with eosin (magnification $\times 100$ ). 
(Figure 7 (b), (c) and (d)). At day 7, HSMCs grew into cell sub-monolayer on the surface of PP and SPP films and secreted the extracellular matrix (ECM) to fill up the holes present in the PHBHHx films (Figure 7 (c') and (d')), while the HSMCs on the P and SP films show a lower cell confluence and less ECM secretion (Figure 7 (a') and (b')). Similarly, Kim et al found that the human bone marrow stromal cells proliferated well to a larger area when attached to the hydrophilic surfaces than those of the hydrophobic surfaces, resulting in the formation of a more flatten morphology [30]. Also Lampin et al. [29] reported that the increase in the surface roughness of poly (methyl methacrylate) film in extent allowed the expansion of the migration of the chick embryo vascular and corneal cells and triggered the subconfluent cells to secrete the extracellular proteins. Accordingly, in our work, the plasma-treated PHBHHx film improves the roughness (shown in Figure 2) and hydrophilicity (shown in Figure 3), leading to the larger amount of ECM secretion and the better cell migration.

\section{CONCLUSION}

In this work, PHBHHx films were irradiated by the low temperature atmospheric plasma for 6 min, resulting in
Day 3

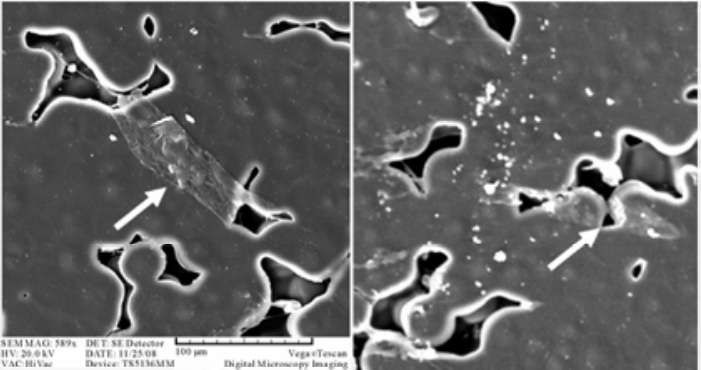

PHBHHx (P)

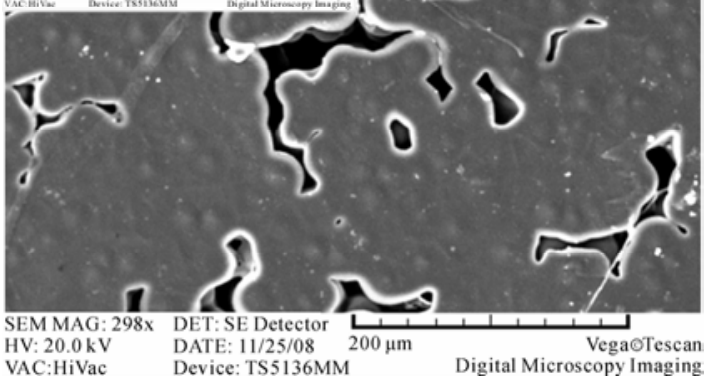

(a)

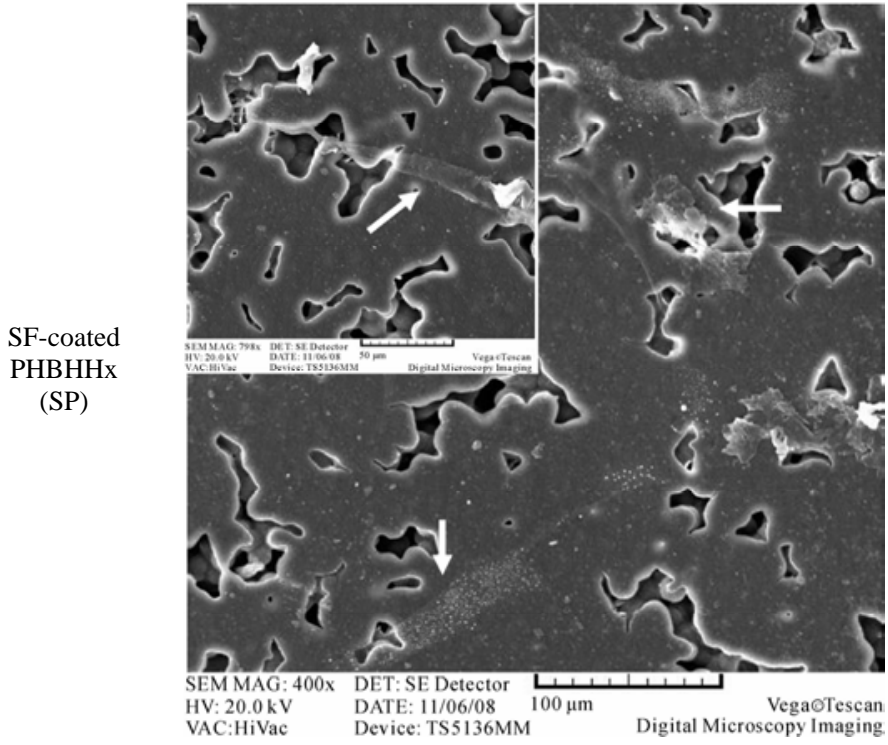

(b)
Day 7

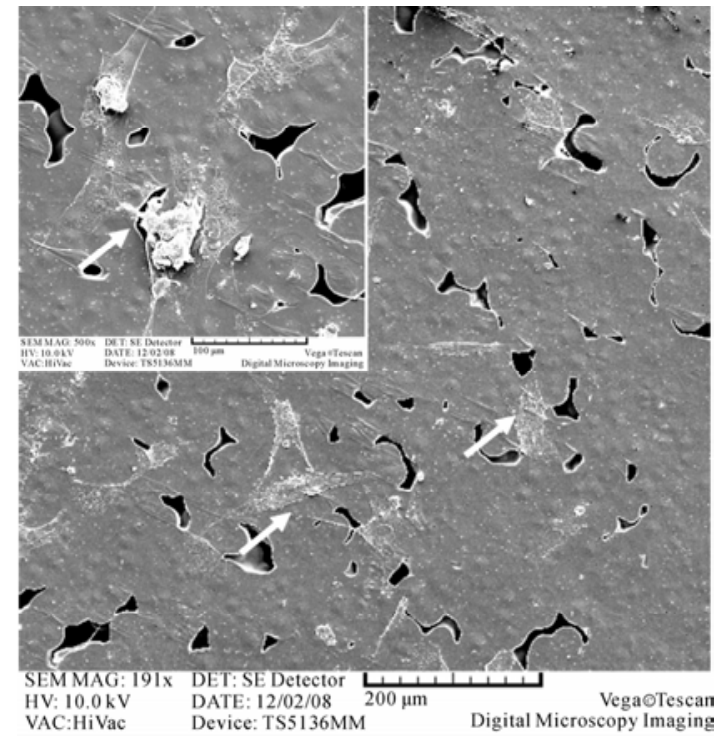

(a')

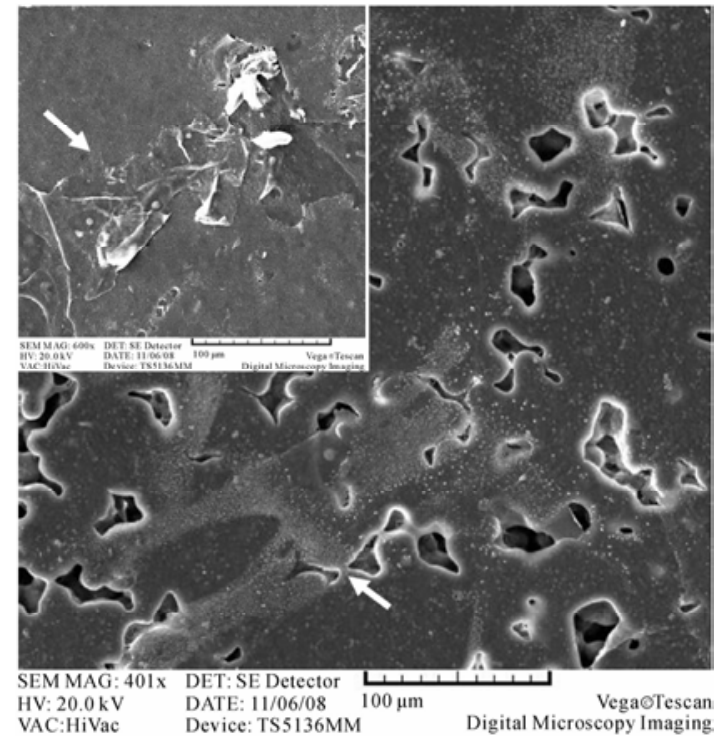

(b’) 


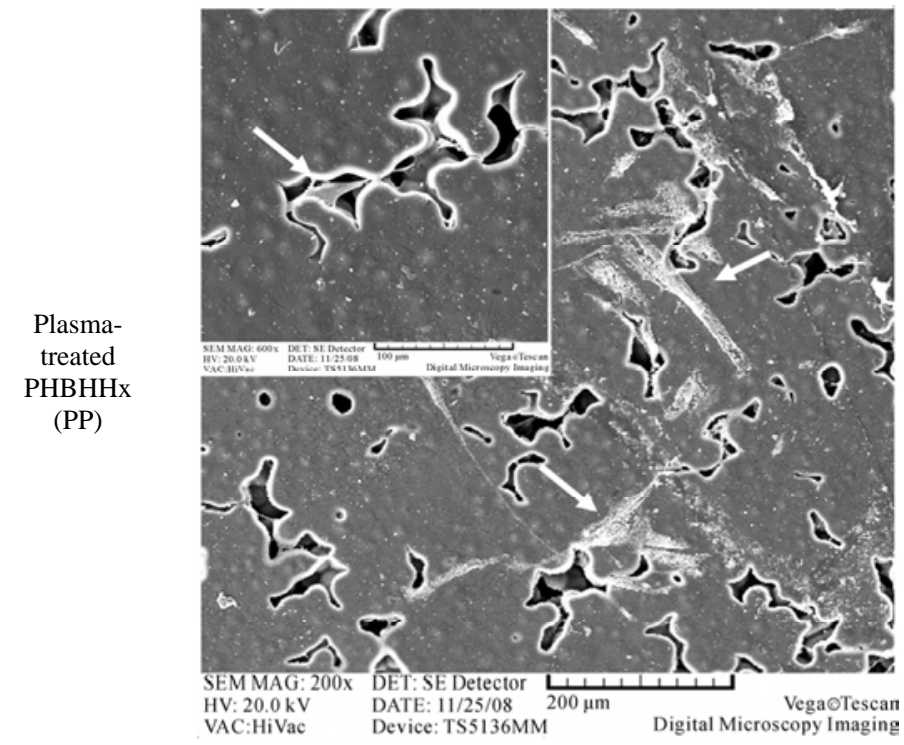

(c)

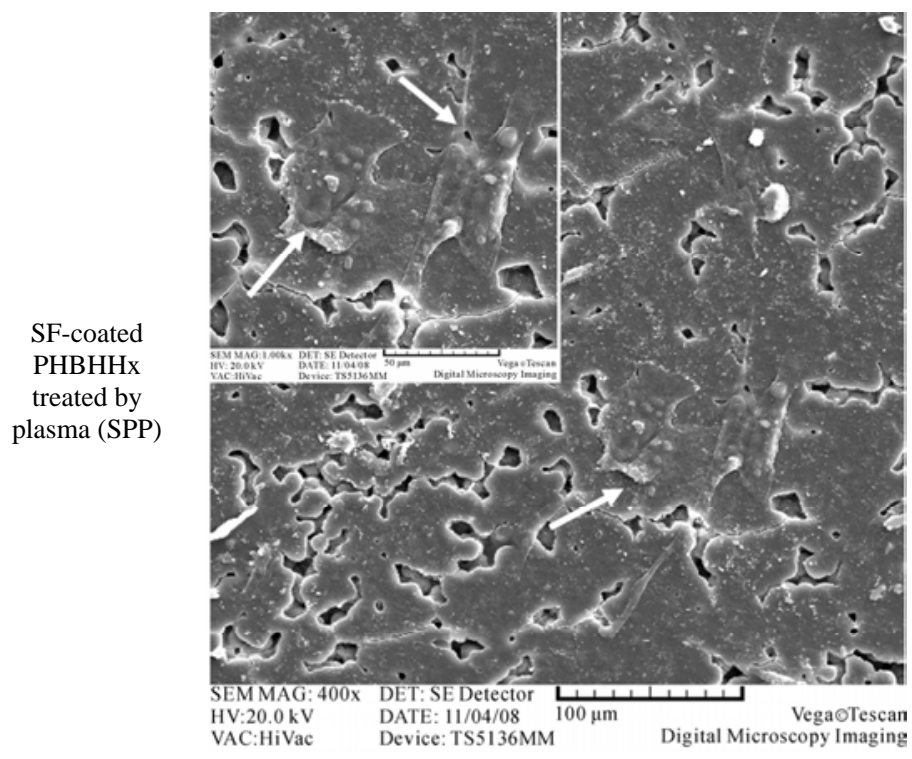

(d)

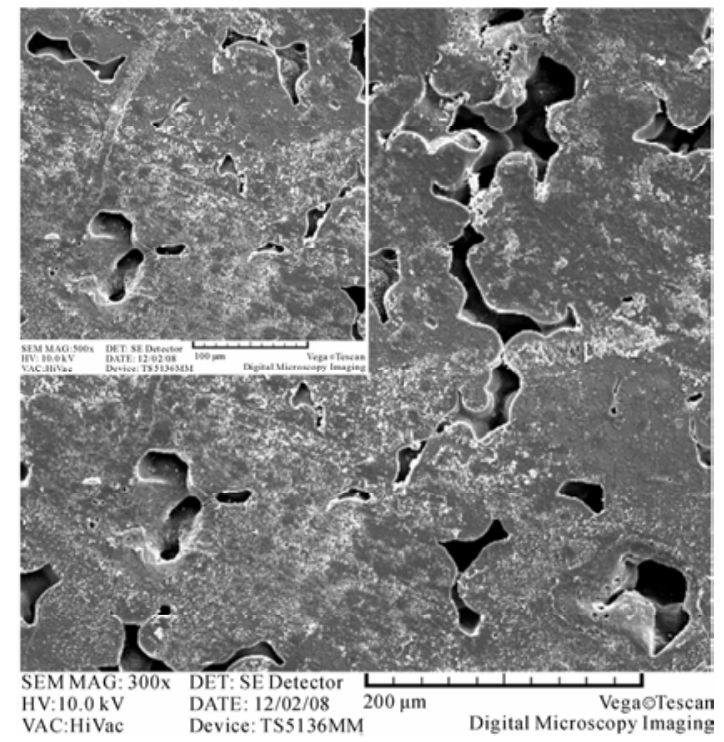

(c')

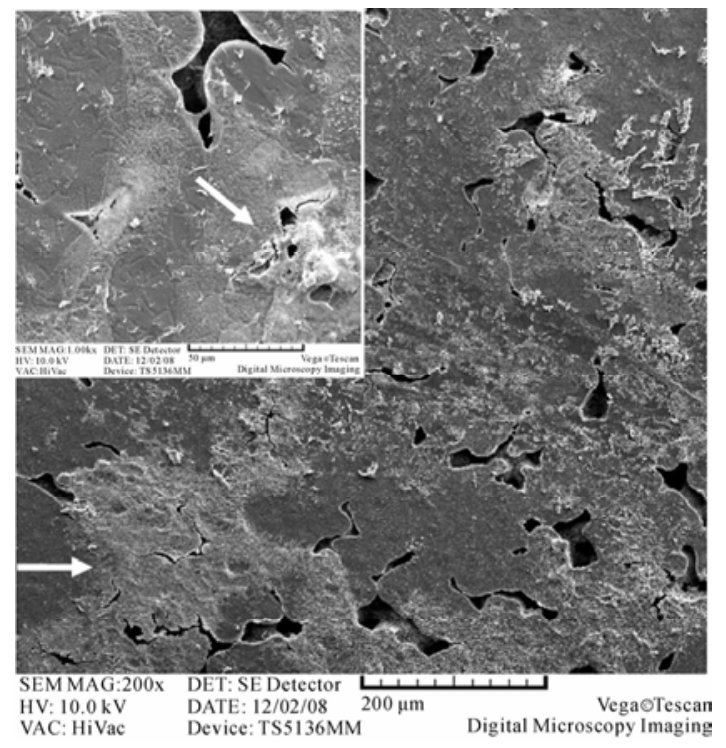

(d')

Figure 7. The morphologies of HSMCs cultured on four types of films: (a) and (a') cells cultured on the pristine PHBHHx film (P) at day 3 and 7; (b) and (b') cells cultured on the SF-coated PHBHHx film (SP) at day 3 and 7; (c) and (c') cells cultured on the plasma treated PHBHHx film (PP) at day 3 and 7; (d) and (d') cells cultured on the SF-coated PHBHHx film treated by plasma (SPP) at day 3 and 7. Arrows point the positions of HSMCs.

an increased roughness and improved hydrophilic surface with low water contact angle. The SF-coated PHBHHx films treated by the plasma were flushed by PBS buffer under the rate of physiological blood flow, proving that SF on the plasma-treated surface have better immobilization strength than that on the surface without plasma treatment. A significant increase in the proliferations of HSMCs is present on the SF-coated PHBHHx films with plasma treatment, and the cell sub-monolayer and the secreted ECM are also formed well on these films.

\section{ACKNOWLEDGEMENTS}

We thank Professor Guoqiang Chen in Tsinghua University for kindly donating the PHBHHx compound. We also thank Mr. Lingxiang Wu in Fudan University for providing the DBD plasma generator. 


\section{REFERENCES}

[1] Armentano, I. (2009) Role of PLLA plasma surface modification in the interaction with human marrow stromal cells. Journal of Applied Polymer Science, 114, 3602-3611.

[2] Chen, G., Zhou, P., Mei, N., Chen, X., Shao, Z.Z., Pan, L.F. and Wu, C.G. (2004) Silk fibroin modified porous poly (E-caprolactone) scaffold for human fibroblast culture in vitro. Journal of Materials Science-Materials in Medicine, 15, 671-677.

[3] Chen, G.Q. and Wu, Q. (2005) The application of polyhydroxyalkanoates as tissue engineering materials. Biomaterials, 26, 6565-6578.

[4] Dumitrascu, N., Borcia C. and Borcia G. (2008) Control of the blood-polymer interface by plasma treatment. Journal of Biomedical Materials Research Part B-Applied Biomaterials, 87B, 364-373.

[5] Dumitrascu, N., Borcia, G., Apetroaei, N. and Popa, G. (2002) Roughness modification of surfaces treated by a pulsed dielectric barrier discharge. Plasma Sources Science \& Technology, 11, 127-134.

[6] Hersel, U., Dahmen, C. and Kessler, H. (2003) RGD modified polymers: biomaterials for stimulated cell adhesion and beyond. Biomaterials, 24, 4385-4415.

[7] Hoerstrup, S.P. (2000) Functional living trileaflet heart valves grown in vitro. Circulation, 102, 44-49.

[8] Khang, G., Kim, S.W., Cho, J.C., Rhee, J.M., Yoon, S.C. and Lee, H.B. (2001) Preparation and characterization of poly (3-hy-droxybutyrate-co-3-hydroxyvalerate) microspheres for the sustained release of 5-fluorouracil. BioMedical Materials and Engineering, 11, 89-103.

[9] Kim, M.S., Shin, Y.N., Cho, M.H., Kim, S.H., Kim, S.K., Cho, Y.H., Khang, G., Lee, I.W. and Lee, H.B. (2007) Adhesion behavior of human bone marrow stromal cells on differentially wettable polymer surfaces. Tissue Engineering, 13, 2095-2103.

[10] Lampin, M., WarocquierClerout, R., Legris, C, Degrange, M. and SigotLuizard, M.F. (1997) Correlation between substratum roughness and wettability, cell adhesion, and cell migration. Journal of Biomedical Materials Research, 36, 99-108.

[11] Langer, R. and Vacanti, J.P. (1993) Tissue Engineering. Science, 260, 920-926.

[12] Lee, S.Y. (1996) Bacterial polyhydroxyalkanoates. Biotechnology and Bioengineering, 49, 1-14.

[13] Li, X.T., Zhang, Y. and Chen, G.Q. (2008a) Nanofibrous polyhy- droxyalkanoate matrices as cell growth supporting materials. Biomaterials, 29, 3720-3728.

[14] Li, X.T., Sun, J., Chen, S. and Chen, G.Q. (2008b) In vitro investigation of maleated poly (3-hydroxybutyrateco-3-hydroxyhexanoate) for its biocompatibility to mouse fibroblast L929 and human microvascular endothelial cells. Journal of Biomedical Materials Research Part A, 87A, 832-842.

[15] Li, Z.G., Lin, H., Ishii, N., Chen, G.Q. and Inoue, Y. (2007) Study of enzymatic degradation of microbial copolyesters consisting of 3-hydroxybutyrate and mdiumchain-length 3-hydroxyalkanoates. Polymer Degradation and Stability, 92, 1708-1714.
[16] Mei, N., Chen, G., Zhou, P., Chen, X., Shao, Z.Z., Pan, L.F. and Wu C.G. (2005) Biocompatibility of poly (epsilon-caprolac-tone) scaffold modified by chitosan - The fibroblasts proliferation in vitro. Journal of Biomaterials Applications, 19, 323-339.

[17] Mei, N., Zhou, P., Pan, L.F., Chen, G., Wu, C.G., Chen, X., Shao, Z.Z. and Chen, G.Q. (2006) Biocompatibility of poly (3-hy-droxybutyrate-co-3-hydroxyhexanoate) modified by silk fibroin. Journal of Materials Science- Materials in Medicine, 17, 749-758.

[18] Niklason, L.E., Gao, J., Abbott, W.M., Hirschi, K.K., Houser, S., Marini, R. and Langer, R. (1999) Functional arteries grown in vitro. Science, 284, 489-493.

[19] Ostrikov, K. and Murphy, A.B. (2007) Plasma-aided nanofabrication: Where is the cutting edge? Journal of Physics D-Applied Physics, 40, 2223-2241.

[20] Robinson, D.E., Marson, A., Short, R.D., Buttle, D.J., Day, A.J., Parry, K.L., Wiles, M., Highfield, P., Mistry, A. and Whittle, J.D. (2008) Surface gradient of functional heparin. Advanced Materials, 20, 1166-1169.

[21] Shangguan, Y.Y., Wang, Y.W., Wu, Q. and Chen, G.Q. (2006) The mechanical properties and in vitro biodegradation and biocompatibility of UV-treated poly (3-hydroxybutyrate-co-3-hydroxyhexanoate). Biomaterials, 27, 2349-2357.

[22] Shen, H., Hu, X.X., Bei, J.Z., Wang, S.G. (2008) The immobilization of basic fibroblast growth factor on plasma-treated poly (lactide-co-glycolide). Biomaterials, 29, 2388-2399.

[23] Shen, H., Hu, X.X., Yang, F., Bel, J.Z. and Wang, S.G. (2007) Combining oxygen plasma treatment with anchorage of cationized gelatin for enhancing cell affinity of poly (lactide-co-glycolide). Biomaterials, 28, 4219-4230.

[24] Shishatskaya, E.I., Voinova, O.N., Goreva, A.V., Mogilnaya, O.A. and Volova, T.G. (2008) Biocompatibility of polyhydroxybutyrate microspheres: in vitro and in vivo evaluation. Journal of Materials Science-Materials in Medicine, 19, 2493-2502.

[25] Siow, K.S., Britcher, L., Kumar, S. and Griesser, H.J. (2006) Plasma methods for the generation of chemically reactive surfaces for biomolecule immobilization and cell colonization - A review. Plasma Processes and Polymers, 3, 392-418.

[26] Sodian, R., Hoerstrup, S.P., Sperling, J.S., Daebritz, S., Martin, D.P., Moran, A.M., Kim, B.S., Schoen, F.J., Vacanti, J.P. and Mayer, J.E. (2000) Early in vivo experience with tissue-engineered trileaflet heart valves. Circulation, 102, 22-29.

[27] Sun, M., Zhou, P., Pan, L.F., Liu, S. and Yang, H.X. (2009) Enhanced cell affinity of the silk fibroin-modified PHBHHx material. Journal of Materials Science-Materials in Medicine, 20, 1743-1751.

[28] Tamada, Y. and Ikada, Y. (1993) Cell-adhesion to plasma-treated polymer surfaces. Polymer, 34, 2208-2212.

[29] Wang, Y.W., Wu, Q., Chen, J.C. and Chen, G.Q. (2005) Evaluation of three-dimensional scaffolds made of blends of hydroxyapatite and poly (3-hydroxybutyrate-co-3hydroxy-hexanoate) for bone reconstruction. Biomaterials, 26, 899-904.

[30] Wu, Q., Wang, Y. and Chen, G.Q. (2009) Medical Application of Microbial Biopolyesters Polyhydroxyalkanoates. Artificial Cells Blood Substitutes and Biotech- 
nology, 37, 1-12.

[31] Xin, X.J., Hussain, M. and Mao, J.J. (2007) Continuing different- tiation of human mesenchymal stem cells and induced chondrogenic and osteogenic lineages in electrospun PLGA nanofiber scaffold. Biomaterials, 28, 316-325.

[32] Yang, M., Zhu, S.S., Chen, Y., Chang, Z.J., Chen, G.Q., Gong, Y.D., Zhao, N.M. and Zhang, X.F. (2004) Studies on bone marrow stromal cells affinity of poly (3- hydroxybutyrate-co-3-hydroxyhexanoate). Biomaterials, 25, 1365-1373.

[33] Zhang, D.M., Cui, F.Z., Luo, Z.S., Lin, Y.B., Zhao, K. and Chen, G.Q. (2000) Wettability improvement of bac- terial polyhy-droxyalkanoates via ion implantation. Surface \& Coatings Technology, 131, 350-354.

[34] Zhang, J.C., Wu, L.B., Jing, D.Y. and Ding, J.D. (2005) A comparative study of porous scaffolds with cubic and spherical macropores. Polymer, 46, 4979-4985.

[35] Zhang, Y., Zhou, P., Pan, L.F., Xie, S.Z., Sun, M. and Li, W.T. (2007) Growth of human smooth muscle cells on the silk fibroin modified-polyhydroxyalkanoate scaffold. Acta Chimica Sinica, 65, 2935-2940.

[36] Zhao, K., Deng, Y., Chen, J.C. and Chen, G.Q. (2003) Polyhy-droxyalkanoate (PHA) scaffolds with good mechanical properties and biocompatibility. Biomaterials, 24, 1041-1045. 